\title{
Evaluation of GeneXpert PA assay compared to genomic and (semi-)quantitative culture methods for direct detection of Pseudomonas aeruginosa in endotracheal aspirates
}

Thomas Ewout van der Schalk1, Jasmine Coppens ${ }^{1,2}$, Leen Timbermont ${ }^{1}$, Agata Turlej-Rogacka ${ }^{1}$, Liesbet Van Heirstraeten ${ }^{1}$, Matilda Berkell ${ }^{1}$, Li Yu ${ }^{4}$, Christine Lammens ${ }^{1}$, Basil Britto Xavier ${ }^{1}$, Veerle Matheeussen ${ }^{2}$, Margareta leven ${ }^{1,2}$, Michael McCarthy ${ }^{5}$, Philippe G. Jorens ${ }^{6}$, Alexey Ruzin³, MarkT. Esser ${ }^{3}$, Samir Kumar-Singh ${ }^{1,7}$, Herman Goossens ${ }^{1,2}$ and Surbhi Malhotra-Kumar ${ }^{1 *}$ (i)

\begin{abstract}
Introduction: Pseudomonas aeruginosa is a common cause of ventilator-associated pneumonia (VAP). Rapid and accurate detection of lower respiratory tract colonization and/or infection with P. aeruginosa may advise targeted preventive (antibody-based) strategies and antibiotic therapy. To investigate this, we compared semi-quantitative culture results from 80 endotracheal aspirates (ETA) collected from mechanically-ventilated patients, to two culture and two non-culture-based methods for detection of P. aeruginosa.

Methods: P. aeruginosa-positive $(n=40)$ and -negative $(n=40)$ ETAs from mechanically ventilated patients analyzed initally by (i) routine semi-quantitative culture, were further analyzed with (ii) quantitative culture on chromogenic ChromID P. aeruginosa and blood agar; (iii) enrichment in brain heart infusion broth followed by plating on blood agar and ChromID P. aeruginosa; (iv) O-antigen acetylase gene-based TaqMan qPCR; and (v) GeneXpert PA PCR assay.
\end{abstract}

Results: Of the 80 ETA samples included, one sample that was negative for $P$. aeruginosa by semi-quantitative culture was found to be positive by the other four methods, and was included in an "extended" gold standard panel. Based on this extended gold standard, both semi-quantitative culture and the GeneXpert PA assay showed $97.6 \%$ sensitivity and $100 \%$ specificity. The quantitative culture, enrichment culture and O-antigen acetylase gene-based TaqMan qPCR had a sensitivity of $97.6 \%, 89.5 \%, 92.7 \%$, and a specificity of $97.4 \%, 100 \%$, and $71.1 \%$, respectively.

Conclusion: This first evaluation of the GeneXpert PA assay with ETA samples found it to be as sensitive and specific as the routine, hospital-based semi-quantitative culture method. Additionally, the GeneXpert PA assay is easy to perform (hands-on time $\approx 5 \mathrm{~min}$ ) and rapid $(\approx 55 \mathrm{~min}$ assay time). The combination of the high sensitivity and high specificity together with the rapid acquisition of results makes the GeneXpert PA assay a highly recommended screening technique. Where this equipment is not available, semi-quantitative culture remains the most sensitive of the culture methods evaluated here for P. aeruginosa detection in ETA samples.

*Correspondence: surbhi.malhotra@uantwerpen.be

${ }^{1}$ Laboratory of Medical Microbiology, Vaccine and Infectious Disease

Institute, University of Antwerp, Wilrijk, Belgium

Full list of author information is available at the end of the article

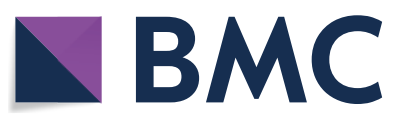

(c) The Author(s) 2021. Open Access This article is licensed under a Creative Commons Attribution 4.0 International License, which permits use, sharing, adaptation, distribution and reproduction in any medium or format, as long as you give appropriate credit to the original author(s) and the source, provide a link to the Creative Commons licence, and indicate if changes were made. The images or other third party material in this article are included in the article's Creative Commons licence, unless indicated otherwise in a credit line to the material. If material is not included in the article's Creative Commons licence and your intended use is not permitted by statutory regulation or exceeds the permitted use, you will need to obtain permission directly from the copyright holder. To view a copy of this licence, visit http://creativecommons.org/licenses/by/4.0/. The Creative Commons Public Domain Dedication waiver (http://creativeco mmons.org/publicdomain/zero/1.0/) applies to the data made available in this article, unless otherwise stated in a credit line to the data. 
Keywords: Cepheid, GeneXpert, Real-time PCR, Rapid diagnostics, VAP, Ventilator-associated pneumonia, ChromID ${ }^{\circledR}$ P. aeruginosa, Chromogenic medium, Pseudomonas aeruginosa

\section{Background}

Pseudomonas aeruginosa infections primarily affect immunocompromised patients and this opportunistic pathogen frequently harbours multiple antibiotic resistance mechanisms [1]. P. aeruginosa is also a frequently-occurring nosocomial pathogen causing lifethreatening infections such as ventilator-associated pneumonia (VAP) in the critically ill patient necessitating ventilation [2]. VAP occurs after endotracheal intubation and is estimated to affect up to $30 \%$ of mechanically ventilated patients [3]. In addition to increasing patient morbidity and mortality, VAP is associated with prolonged hospital stay and increased healthcare costs, and is classically detected based on clinical signs, a new infiltrate on chest X-rays and, importantly, detection of the causative pathogen in respiratory samples $[3,4]$.

Bacterial colonization is a well-known risk factor for VAP [5]. Therefore, identification of colonizing pathogens by surveillance cultures is employed as part of a preemptive strategy for VAP. Because endotracheal aspirates (ETAs) can be acquired easily from intubated patients with limited complications, collection of ETAs represents a relatively non-invasive procedure and these samples are commonly used for surveillance microbiological cultures [6].

While culture of pathogens remains the gold standard, molecular tests that typically have a shorter turnaround time can drastically decrease the critical time-to-initiation of preventive and therapeutic strategies, including the initiation of the appropriate antibacterial treatment. In this study, we evaluated the current research use only GeneXpert PA assay, which directly detects P. aeruginosa from ETA samples, and compared it to culture and nonculture based methods for detection of $P$. aeruginosa in ETA samples.

\section{Materials and methods}

\section{Study design and sample collection}

The study was designed to assess the performance of a rapid screening test, the Cepheid GeneXpert PA assay, in patients at risk of developing VAP. During February 2017-September 2018, ETA samples were collected from mechanically ventilated adult patients admitted to the intensive care unit (ICU) at the Antwerp University Hospital (UZA), either for surveillance cultures or as routine samples obtained in patients with a suspected (pulmonary) infection (Fig. 1). Clinical patient data regarding the development of VAP was also collected in the patient data management system (Metavision, iMD soft). VAP was diagnosed by a board certified senior ICU physician according to the most stringent and "validated" definition: a new bacterial pneumonia present in patients receiving mechanical ventilation for at least $48 \mathrm{~h}$ as characterised by a new infiltrate on the chest radiograph, signs of infection and detection of a bacterial causative agent [7], the latter most using semi-quantitative culture results as part of routine clinical practice. ETAs were analysed immediately at the UZA Clinical Microbiology Laboratory using quadrant-based, semi-quantitative culture on blood agar, chocolate agar, McConkey, and colistinnalidixic acid agar (CNA, Oxoid, the UK) where bacterial growth was evaluated after $24 \mathrm{~h}$ of incubation [8]. Sample inclusion in the study was based on semi-quantitative culture results from the UZA Clinical Microbiology Laboratory for routine diagnosis of VAP. Based on the quadrant growth, the semi-quantitative culture method categorizes positive samples as light (growth in quadrant one), moderate (growth in quadrants two and three) and heavy (growth in all four quadrants). If positive, the sample was included in this study with the corresponding classification (Fig. 1). Following a P. aeruginosa-positive sample, the first subsequent semi-quantitative culturenegative sample was included in the negative group (Fig. 1). At the end of the study period, 80 samples were collected consisting of 40 each where $P$. aeruginosa was either detected or not. Among the P. aeruginosa-positive samples, 20,10, and 10 were classified as light, moderate, and heavy $P$. aeruginosa loads based on semi-quantitative culture, respectively (Fig. 1, Additional file 1: Table S1). Collected ETA samples were stored at $4{ }^{\circ} \mathrm{C}$ for a maximum of $48 \mathrm{~h}$. Upon inclusion in the study, they were further processed with different methods for $P$. aeruginosa detection and quantitation at the Laboratory of Medical Microbiology, University of Antwerp. Methods used were: GeneXpert PA assay; quantitative culture on chromogenic ChromID ${ }^{\circledR}$ P. aeruginosa and blood agar; brain heart infusion (BHI) broth enrichment followed by blood agar and ChromID ${ }^{\circledR}$ P. aeruginosa plating; and quantitative TaqMan real-time PCR (qPCR) targeting the O-antigen acetylase on extracted DNA. The study was approved by the UZA ethics committee (Belgian registration number B300201629199).

\section{GeneXpert ${ }^{\circledR} \mathrm{PA}$ assay}

The GeneXpert ${ }^{\circledR}$ PA assay (Cepheid, USA) is a PCR test that is currently available for research use. The assay was 


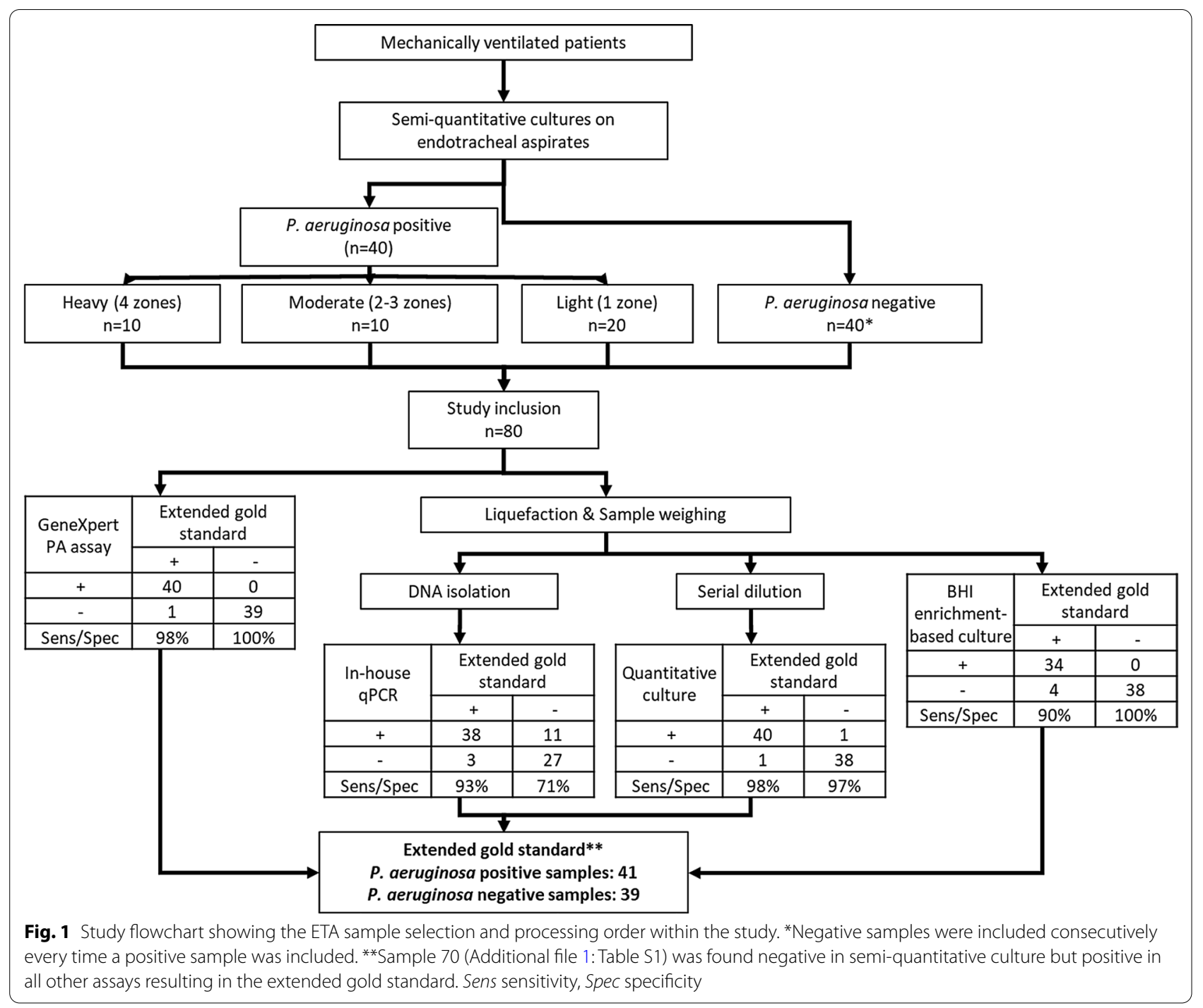

performed according to the manufacturer's instructions. Briefly, ETA samples were adsorbed onto a Cepheid collection device swab (CLASSIQ, COPAN), dissolved in elution buffer and vortexed at high speed for $10 \mathrm{~s}$ (Scientific Industries Inc., USA). From the elution reagent, $1.7 \mathrm{ml}$ was transferred to the cartridge and analysed by GeneXpert ${ }^{\circledR}$ Dx system v4.7b (Cepheid, USA). The overall process of extraction, amplification, and detection of intact bacterial cells was completed in $55 \mathrm{~min}$. Samples were reported by the accompanied GeneXpert software as either P. aeruginosa "detected" or "not detected". The detection was based on the P. aeruginosa PCR cycle threshold $(\mathrm{Ct})$ values and the $\mathrm{Ct}$ values of the sample adequacy control (a multiplexed control that contains primers and probes for the detection of human cells or DNA and should only be considerd in case of a
$P$. aeruginosa-negative result) and sample processing control (a control containing Bacillus globigii spores for verification of successful bacterial cell lysis) with valid $\mathrm{Ct}$ ranges between 3 and 45 for all three targets.

\section{Sample preparation for comparator culture-based and qPCR methods}

After initiating the GeneXpert assay, the remaining ETA sample was homogenised by blending and liquefaction. All samples were blended with the dispersing instrument T10 basic ULTRA-TURRAX (IKA, Staufen, Germany) for $10 \mathrm{~s}$ at maximum speed on ice. Depending on the viscosity of the sample, checked by visual inspection, the blending time was increased by steps of $10 \mathrm{~s}$, with a maximum blending time of $60 \mathrm{~s}$. After blending, the samples were liquefied with lysomucil ( $10 \% N$-acetylcysteine, 
Zambon, Milan, Italy). Three ml lysomucil was dissolved in $12 \mathrm{ml}$ phosphate-buffered saline, and an equal amount in volume $(\approx 300 \mu \mathrm{l})$ of liquefying reagent was added to the sample, and vortexed at full speed for $10 \mathrm{~s}$. After incubation at $37^{\circ} \mathrm{C}$ for $15 \mathrm{~min}$, the samples were vortexed again at full speed for $10 \mathrm{~s}$, and the incubation step was repeated. The sample was then split up in $\approx 400 \mu \mathrm{l}$ for quantitative culture and enrichment-based culture, and $\approx 200 \mu \mathrm{l}$ for O-antigen acetylase gene-based qRT-PCR.

\section{Quantitative cultures}

Serial dilutions of the liquefied samples were spirally-plated (Eddy Jet, program 6; $50 \mu \mathrm{l}$ logarithmic spreading; IUL, Spain) on chromogenic ChromID $^{\circledR} P$. aeruginosa medium (bioMerieux, France) and on blood agar (Columbia II Agar Base, Oxoid) with 5\% defibrinated horse blood. After $24 \mathrm{~h}$ of incubation, violet to orange $P$. aeruginosa colonies on the ChromID ${ }^{\circledR} P$. aeruginosa were counted and when no growth of $P$. aeruginosa was detected, the blood agar plate was screened for presence of $P$. aeruginosa. At least one presumptive $P$. aeruginosa colony per sample was speciated by MALDITOF (Bruker, USA) and P. aeruginosa loads were calculated as colony-forming units (CFU)/ml for each sample. Limit of detection was $40 \mathrm{CFU} / \mathrm{ml}$ with this method on both blood and ChromID ${ }^{\circledR}$ P. aeruginosa agar.

\section{Enrichment-based cultures}

Additional enrichment was performed by overnight incubation of a small leftover volume $(\approx 100 \mu \mathrm{l})$ of the liquefied sample in BHI broth followed by plating on ChromID ${ }^{\circledR}$ P. aeruginosa as well as on blood agar plates. Presumptive $P$. aeruginosa colonies were confirmed by MALDI-TOF (Bruker, USA).

\section{O-antigen acetylase gene-based qRT-PCR}

$200 \mu \mathrm{l}$ of the liquefied sample was subjected to proteinase $\mathrm{K}$ treatment for $15 \mathrm{~min}$ at $56{ }^{\circ} \mathrm{C}$ followed by automated DNA extraction (NucliSENS $^{\circledR}$ EasyMag $^{\circledR}$, bioMérieux SA, France) and frozen until batch analysis by an inhouse developed qPCR assay. Concentrations of $P$. aeruginosa DNA in samples were determined using $\mathrm{qPCR}$ targeting the $\mathrm{O}$-antigen acetylase gene using previously designed primers [9] in combination with an in-house developed Taqman probe. The assay was performed in a $20 \mu \mathrm{l}$ reaction volume containing $10 \mu \mathrm{l}$ of $2 \mathrm{x}$ SensiFAST $^{\mathrm{TM}}$ Probe No-ROX Kit (Bioline, London, UK), 400 nM concentrations of primers PA431CF (CTGGGTCGAAAG GTGGTTGTTATC) and PA431CR (GCGGCTGGT GCGGCTGAGTC), and $150 \mathrm{nM}$ TaqMan probe (cy5CGAACAGCGCATTCACGTAGG-BBQ) together with $4 \mu \mathrm{l}$ of DNA template. Amplification was carried out on the CFX96 Touch $^{\text {TM }}$ Real-Time PCR detection system
(Bio-Rad Laboratories Inc, California, USA) using the following cycling parameters: $5 \mathrm{~min}$ at $95{ }^{\circ} \mathrm{C}$ and 40 cycles of $10 \mathrm{~s}$ at $95{ }^{\circ} \mathrm{C}$ and $50 \mathrm{~s}$ at $60^{\circ} \mathrm{C}$. Bacterial loads were calculated based on a standard curve that was set up using Avogadro's constant and the molecular weight of serially diluted $\mathrm{O}$-antigen acetylase gene PCR product from P. aeruginosa ATCC27853 [10]. Samples and standard curves were run in triplicate and were considered valid when at least two out of the three replicates had a $\mathrm{Ct}$ with less than 0.2 difference. The qPCR assay was validated with $P$. aeruginosa-negative ETAs spiked with different concentrations of $P$. aeruginosa before utilization in this study (data not shown).

\section{Statistical analysis}

Normality was assessed using Shapiro's test followed by statistical comparisons of patient groups stratified by clinical outcome using either ANOVA or Kruskal-Wallis with two-tailed t-test or Mann-Whitney posthoc testing as indicated. Correlation between $\mathrm{Ct}$ values of the GeneXpert PA assay and the in-house qPCR and the relation between Ct values of both PCR assays and CFU/ $\mathrm{ml}$ from the quantitative culture were assessed by linear regression by an F-test as well as by Pearson's correlation coefficient.

Receiver Operator Characteristic analysis was performed in order to assess the GeneXpert PA assay as a standalone diagnostic for $P$. aeruginosa VAP where the 95\% confidence interval was determined using the Wilson/Brown hybrid method.

\section{Results}

We analysed 80 ETA samples utilizing semi-quantitative culture, GeneXpert assay, quantitative culture, enrichment-culture, and $\mathrm{O}$-antigen acetylase gene-based qPCR (overview of results in Additional file 1: Table S1). Sample 70 was $P$. aeruginosa-negative by semi-quantitative culture while $P$. aeruginosa-positive by all the other methods, albeit with low $P$. aeruginosa loads by quantitative culture $\left(2.3 \times 10^{4}(1.4 \%\right.$ of total growth) $)$ and high $\mathrm{Ct}$ values by the in-house qPCR $(\mathrm{Ct}=30.1)$. Sample 70 was thus considered positive which resulted in an extended gold standard with $41 P$. aeruginosa-positive samples and $39 P$. aeruginosa-negative samples.

\section{GeneXpert PA assay is an accurate method for detection of $P$. aeruginosa in ETA samples}

With the extended gold standard panel as a comparator, the GeneXpert PA assay showed $97.6 \%$ sensitivity (95\% CI $87.1-99.9 \%)$ and $100 \%$ specificity (95\% CI $91.0-100 \%$, Table 1). The semi-quantitative culture, quantitative culture, and GeneXpert PA assay showed the highest sensitivity with $97.6 \%$. The sensitivity was not $100 \%$ since the 
Table 1 Analytical performance of the five methods utilized for P. aeruginosa detection from ETA samples compared to the extended gold standard

\begin{tabular}{|c|c|c|c|c|}
\hline \multirow[t]{2}{*}{ Methods } & \multicolumn{2}{|c|}{$\begin{array}{l}\text { Extended gold } \\
\text { standard }^{\mathrm{a}}\end{array}$} & \multirow{2}{*}{$\begin{array}{l}\text { Sensitivity (95\% } \\
\text { confidence } \\
\text { interval) }\end{array}$} & \multirow{2}{*}{$\begin{array}{l}\text { Specificity ( } 95 \% \\
\text { confidence } \\
\text { interval) }\end{array}$} \\
\hline & $\begin{array}{l}\text { Positive } \\
(n=41)\end{array}$ & $\begin{array}{l}\text { Negative } \\
(n=39)\end{array}$ & & \\
\hline \multicolumn{5}{|c|}{ Quantitative culture } \\
\hline Positive & 40 & 1 & $97.6 \%(87.1-99.9)$ & $97.4 \%(86.5-99.9)$ \\
\hline Negative & 1 & 38 & & \\
\hline \multicolumn{5}{|c|}{ Enrichment culture ( 4 samples could not be tested) } \\
\hline Positive & 34 & 0 & $89.5 \%(75.2-97.1)$ & $100 \%(90.8-100)$ \\
\hline Negative & 4 & 38 & & \\
\hline \multicolumn{5}{|c|}{ In-house qPCR (1 sample could not be tested) } \\
\hline Positive & 38 & 11 & $92.7 \%(80.1-98.5)$ & $71.1 \%(54.1-84.6)$ \\
\hline Negative & 3 & 27 & & \\
\hline \multicolumn{5}{|c|}{ GeneXpert PA assay } \\
\hline Positive & 40 & 0 & $97.6 \%(87.1-99.9)$ & $100 \%(91.0-100)$ \\
\hline Negative & 1 & 39 & & \\
\hline \multicolumn{5}{|c|}{ Semi-quantitative culture } \\
\hline Positive & 40 & 0 & $97.6 \%(87.1-99.9)$ & $100 \%(91.0-100)$ \\
\hline Negative & 1 & 39 & & \\
\hline
\end{tabular}

${ }^{\text {a }}$. aeruginosa detected by semi-quantitative culture plus one sample that showed $P$. aeruginosa presence by the other four methods but not by semiquantitative culture

GeneXpert PA assay had one negative sample (sample 28) that was also negative by qPCR but confirmed to be positive by semi-quantitative culture and quantitative culture with a load of $40 \mathrm{CFU} / \mathrm{ml}$.

\section{Semi-quantitative culture has the highest sensitivity} and specificity among the culture-based techniques tested Next, we compared the three culture-based methods, semi-quantitative culture, quantitative culture and enrichment-culture with the extended gold standard results. Semi-quantitative and quantitative culture both showed similar and higher sensitivities (97.6\%, 95\% CI 87.1-99.9\%) than enrichment-culture $(89.5 \%$, 95\% CI 75.2-97.1\%). The specificities of the semi-quantitative culture and the enrichment-culture was 100\% (95\% CI 91.0-100\% and $90.8-100 \%$, respectively) while for the quantitative culture the specificity was $97.4 \%$ (95\% CI 86.5-99.9\%, Table 1). The single sample (sample 70) that was negative for $P$. aeruginosa by semi-quantitative culture had a low PA load. For this sample, Ct values of both PCR methods were above 30 and the load in quantitative culture was $2.3 \times 10^{4} \mathrm{CFU} / \mathrm{ml}$, less than $3 \%$ compared to the total growth on the blood agar.

Quantitative culture was negative for one sample (sample 41) that was also negative by the enrichment-culture, which indicates a possible heterogeneity of the sample since both the GeneXpert and in-house qPCR detected $P$. aeruginosa. One sample was positive by quantitative culture (sample 63) that was found negative by all other methods where only one $P$. aeruginosa colony was found on blood agar with no growth on the ChromID ${ }^{\circledR}$ P. aeruginosa plate. In order to compare bacterial loads, ratio of $P$. aeruginosa in the total bacterial growth $(\mathrm{CFU} / \mathrm{ml})$ on quantitative culture gave comparable results to semiquantitative culture (Additional file 1: Table S1). Enrichment cultures resulted in 34 positive samples out of the in total 38 P. aeruginosa-positive samples tested and no positives in the $38 P$. aeruginosa-negative samples tested. For four samples (7, 36, 39 and 50, marked N/A in Additional file 1: Table S1), the amount was not enough to process with enrichment culture. These were, however, analysed with the other methods. The four false-negatives of the enrichment-based cultures were samples 3, 25, 26 and 31. The latter three had low loads in the semi-quantitative and quantitative culture $\left(<10^{3} \mathrm{CFU} / \mathrm{ml}\right)$ and the GeneXpert PA assay gave high Ct values (>30). Sample $3 \mathrm{had}$ a high load in semi-quantitative culture and in quantitative culture above $10^{7} \mathrm{CFU} / \mathrm{ml}$. All of these samples were also negative on the blood agar of the enrichment cultures.

\section{In-house qPCR showed high sensitivity for $P$. aeruginosa detection in ETA samples}

The limit of detection of $P$. aeruginosa in ETA by the inhouse qPCR was $\approx 5 \times 10^{2} \mathrm{CFU} / \mathrm{ml}$, and the upper limit of quantification was $>10^{8} \mathrm{CFU} / \mathrm{ml}$ and is in agreement with the diagnostic thresholds for ETA of $\geq 10^{5} \mathrm{CFU} /$ $\mathrm{ml}$ based on quantitative culture [11]. For one sample there was insufficient sample volume for DNA isolation (sample 78). With this qPCR, 49/79 samples tested positive for $P$. aeruginosa and showed a 92.7\% (95\% CI $80.1-89.5 \%$ ) sensitivity and a $71.1 \%$ (95\% CI 54.1$84.6 \%)$ specificity with the extended gold standard as reference (Table 1 ).

Investigation of the correlation of GeneXpert and inhouse qPCR $\mathrm{Ct}$ values of all the $P$. aeruginosa-positive samples showed a Pearson correlation of $0.90(p<0.0001$, Fig. 2A). On average for all the samples, the in-house qPCR had a Ct value that was $5.1 \mathrm{Ct}$ lower than the GeneXpert PA assay. This is most likely because the GeneXpert assay detects only intact $P$. aeruginosa cells due to an extra filtration and washing step that removes extracellular $P$. aeruginosa DNA. There was only one sample (sample 18) that showed a lower $\mathrm{Ct}$ value $(1 \mathrm{Ct})$ in the GeneXpert PA assay than in the in-house qPCR. Ct values of both the GeneXpert and the in-house qPCR compared to the $\log 10$ values of the quantitative culture showed a Pearson correlation of -0.92 and -0.83 $(p<0.0001)$, respectively (Fig. 2B). 


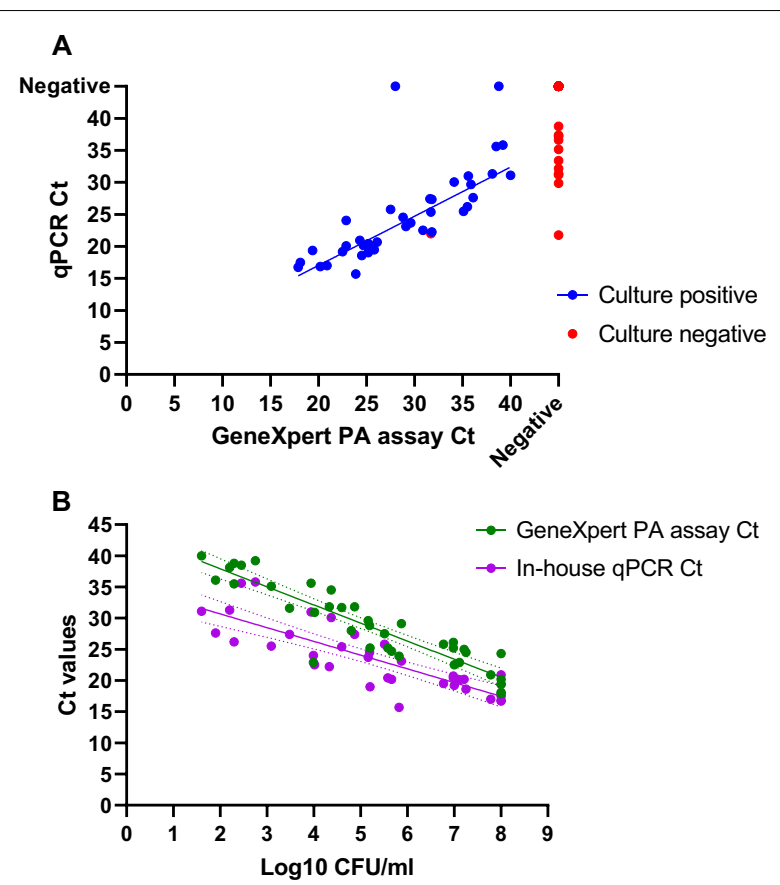

Fig. 2 A Correlation of Ct values between the GeneXpert PA assay (horizontal-axis) and the in-house qPCR (vertical-axis). Each dot represents a sample and is plotted based on the $\mathrm{Ct}$ values from the in-house qPCR (y-axis) and GeneXpert PA assay (x-axis). The line shows the linear regression fit based on the formula: In-house $\mathrm{qPCR} C \mathrm{Ct}=0.7587 \times$ GeneXpert PA Ct +1.821 . Eleven in-house $\mathrm{gPCR}$ Cts and three GeneXpert PA assay Cts were not used to calculate the correlation because they were negative in the other assays. B Correlation of Ct-values of the GeneXpert PA assay (vertical-axis) and the quantitative culture $\log 10$ CFU/ml (horizontal-axis) (green) and the correlation between the in-house RT-qPCR Ct values and the quantitative culture $\log 10 \mathrm{CFU} / \mathrm{ml}$ (purple). The lines in the corresponding colors show the linear regression based on the following formulas. For the GeneXpert PA assay: $\mathrm{Ct}=-2.900 \times \log 10$ $\mathrm{CFU} / \mathrm{ml}+43.72$. The in-house $\mathrm{qPCR} C \mathrm{Ct}=-2.202 \times \log 10 \mathrm{CFU} /$ $\mathrm{ml}+35.08$

\section{Detection of $P$. aeruginosa as a causative agent of VAP}

VAP was diagnosed based on a combination of clinical data and the detection of a causative agent as part of routine clinical practice. Based on clinical data in combination with the semi-quantitative cultures performed at the hospital on 13 samples (11 patients) collected within one day of clinical VAP diagnosis (sample no. 4, 5, 7, 10, $13,14,17,18,20,21,30,31$, and 32), nine patients were assigned as having $P$. aeruginosa VAP. All samples from these 9 patients were found positive with all the methods. There were also 10 patients (all with at least one sample within one day of VAP diagnosis,) diagnosed with non- $P$. aeruginosa VAP (sample no. 6, 15, 19, 22, 25, 26, 28, 34, $35,36,37$, and 39) of which five patients were $P$. aeruginosa-positive although another pathogen was present in higher loads. These results were confirmed in quantitative culture for four of the five patients with loads below $10^{5} \mathrm{CFU} / \mathrm{ml}$. However, ETA from one patient showed an overgrowth of $P$. aeruginosa.

We studied whether the $P$. aeruginosa loads by each of the quantitative assays (except semi-quantitative and enrichment culture that do not have a quantitative output) correlated with VAP diagnosis due to $P$ aeruginosa. Therefore, the samples were split up into four groups: (i) samples from patients with $P$. aeruginosa VAP (PA VAP) ( $\mathrm{n}=13$ samples), (ii) samples from patients with non- $P$. aeruginosa VAP (non-PA VAP) $(\mathrm{n}=12)$, (iii) samples from patients admitted to the ICU with a pneumonia $(n=4)$, and (iv) samples from patients that did not have any pneumonia signs during their stay or at time of admission $(\mathrm{n}=12)$.

The loads of $P$. aeruginosa in samples from PA VAP and non-PA VAP patients were compared. The PA VAP group showed lower $\mathrm{Ct}$ values than the non-PA VAP group in both qPCR assays ( $p>0.05$ for both GeneXpert PA (PA VAP: 26.4, 23.3-29.4 vs. non-PA VAP: 32.2, 26.9-37.4) and for the in-house qPCR (PA VAP: 21.4, 19.6-23.3 vs. non-PA VAP: 32.2, 26.9-37.4) with a two-tailed T-test, Fig. 3A). No significant differences were found for CFU/ $\mathrm{ml}$ counts calculated from the in-house qPCR $\mathrm{Ct}$ values using the linear regression equation (starting quantity mean (calculated in Biorad CFX maestro 1.1 version 4.1.2433.1219) $\times 350 \times 2)$ compared to the quantitative culture CFU/ml (Fig. 3B). However, the proportion of $P$. aeruginosa in the total growth in quantitative culture (\% P. aeruginosa/total growth on blood agar, Fig. 3C) showed a significant difference between the PA VAP and non-PA VAP groups $(p=0.014)$, and a slight difference between the PA VAP and patients that did not develop a pneumonia during their stay $(p=0.07)$. Also no significant differences with any of the three methods were found between $P$. aeruginosa loads in the pre-VAP and the VAP diagnosis samples within the same patient in the four patients where both samples were available.

Finally, we investigated the use of the GeneXpert PA assay as a diagnostic tool for PA VAP using Receiver Operating Characteristic analysis which showed an AUC of 0.69 for samples from patients diagnosed with VAP (95\% confidence interval of 0.46-0.93) (Receiver Operating Characteristic curve, Additional file 1: Figure S1, and Fig. 3A). Although a highly sensitive and specific assay, the fact that $P$. aeruginosa detection in samples does not always correlate to PA VAP, as also seen in our study, makes it difficult for any test based purely on $P$. aeuginosa detection to function as a (standalone) diagnostic for PA VAP. 


\section{Discussion}

Early and reliable screening for $P$. aeruginosa in the lower respiratory tract may inform targeted and novel preventive strategies like antibody-based therapy (e.g. MEDI3902 clinicaltrials.gov: NCT02696902) [12]. With this in mind, we studied and compared two PCR-based and three culture-based methods for $P$. aeruginosa detection in ETA samples, including semi-quantitative culture routinely used in our hospital. In this study, semi-quantitative culture and the GeneXpert PA assay emerged as the most sensitive and specific methods for detection of $P$. aeruginosa in ETA samples. We recently studied and showed high sensitivity and specificity for GeneXpert MRSA/SA ETA assay which directly detects S. aureus from ETA samples compared to the same methods used in this study [13]. The combination of quantitative culture with enrichment culture also resulted in similar sensitivity and specificity as compared to semi-quantitative culture and the GeneXpert PA assay. Nevertheless, with the total hands-on time for preparing the sample of less than 2 min-and with the test completed in less than an hour, the GeneXpert PA assay is a superior method when results need to be received quickly while closely resembling the microbiological diagnosis of semi-quantitative culture. In addition, GeneXpert PA assay is standardized (same method and same equipment used by different laboratories) and therefore should be less affected by crosslaboratory variability than the culture-based methods.

Quantitative culture had a similar sensitivity as the semi-quantitative culture and GeneXpert showing that the pre-treatment with lysomucil was not inhibitory for $P$. aeruginosa recovery. The inhibitory effect of $N$-acetylcysteine on bacterial growth was reported previously [14], however it was not profound in our study as only one sample that had a low load in semi-quantitative culture (non- $N$-acetylcysteine treated) was negative by quantitative culture and enrichment culture ( $N$-acetylcysteine treated). Other possible reasons for this discrepancy between the semi-quantitative and quantitative cultures could be because the former was performed directly on samples and the latter post-sample liquefaction. Also, the use of different types of $P$. aeruginosa identification agar plates with both methods might have impacted detection. It has been shown that the ChomID
P. aeruginosa agar has a slightly lower sensitivity than nalidixic acid-based agar plates [15].

Based on our extended gold standard, the least specific assay was the in-house qPCR where $11 \mathrm{P}$. aeruginosanegative ETA samples tested positive most likely due to detection of extracellular DNA from the lysed $P$. aeruginosa cells. The discrepancies between the quantifications from culture and the molecular methods could be attributed to different factors. For instance, while culture-negative results could arise due to prior antibiotic use (not recorded in the study) or poor sample handling, the ability to detect bacteria at low concentrations by qPCR, also from extracellular DNA, could (wrongly) classify this test as non-specific in comparison to culture or even to the GeneXpert PA assay. Although a qPCR-based assay, the GeneXpert PA does not pose this problem as it detects only intact bacteria through utilization of an additional filtration and washing step that removes extracellular DNA within the cartridge. This is reflected by the generally higher $\mathrm{Ct}$ values of the GeneXpert compared to the in-house qPCR, also making the former assay more comparable with culture-based methods. The combination of only detecting intact bacteria and shorter hands-on time makes the GeneXpert PA assay a valuable and easy tool for $P$. aeruginosa detection in comparison to traditional qPCR assays. It shows the same advantage as a culturebased assay by detecting intact bacteria but has the benefits of being faster with a shorter hands-on time. Thus, it could be an extremely useful assay to screen patients for $P$. aeruginosa colonisation for infection prevention control.

One of the strengths of our study was inclusion of samples from patients that were clinically diagnosed with PA VAP. Of the methods tested in our study, quantitative culture was the most specific in differentiating PA and non-PA VAP. Although in 4 samples from non-PA VAP, the $P$. aeruginosa load was above the PA VAP average (84\% CI 63.8-102\%) underscoring the potential pitfall of utilising $P$. aeruginosa loads to be indicative of VAP.

The fact that quantitative culture which calculates the ratio of $P$. aeruginosa/total growth is still the most accurate assay for detection of PA VAP might be related to prior received empirical treatment with (broad-spectrum) antibiotics that decrease the correlation with

(See figure on next page.)

Fig. 3 A Ct values from GeneXpert PA assay (blue) and in-house GPCR (red) of ETA samples from patients diagnosed with PA VAP, VAP due to other pathogens, pneumonia at admission, or patients without pneumonia. The mean and 95\% confidence interval are shown. B $P$. aeruginosa loads (CFU/ml) in ETA samples from quantitative culture (red), calculated from the in-house qPCR Ct values using the linear regression equation (blue) of ETA samples from patients diagnosed with PA VAP, VAP due to other pathogens, pneumonia at admission, or patients without pneumonia. The mean and 95\% confidence interval are shown. C P. aeruginosa proportions (\% P. aeruginosa/total growth on blood agar, in green) calculated from quantitative culture of ETA samples from patients diagnosed with PA VAP, VAP due to other pathogens, pneumonia at admission, or patients without pneumonia. The mean and 95\% confidence interval are shown.*Indicates a significant difference $(p<0.05)$ between groups 


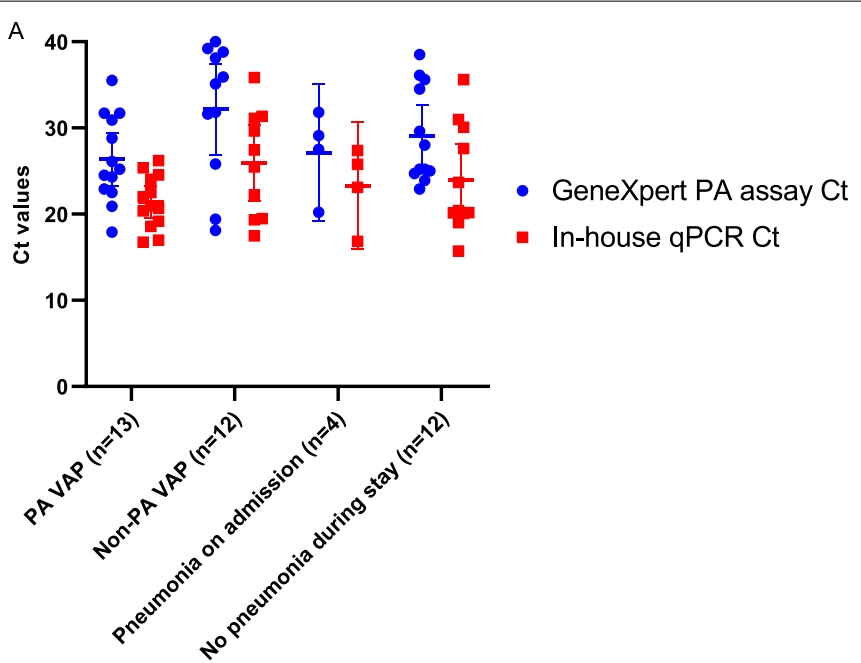

B
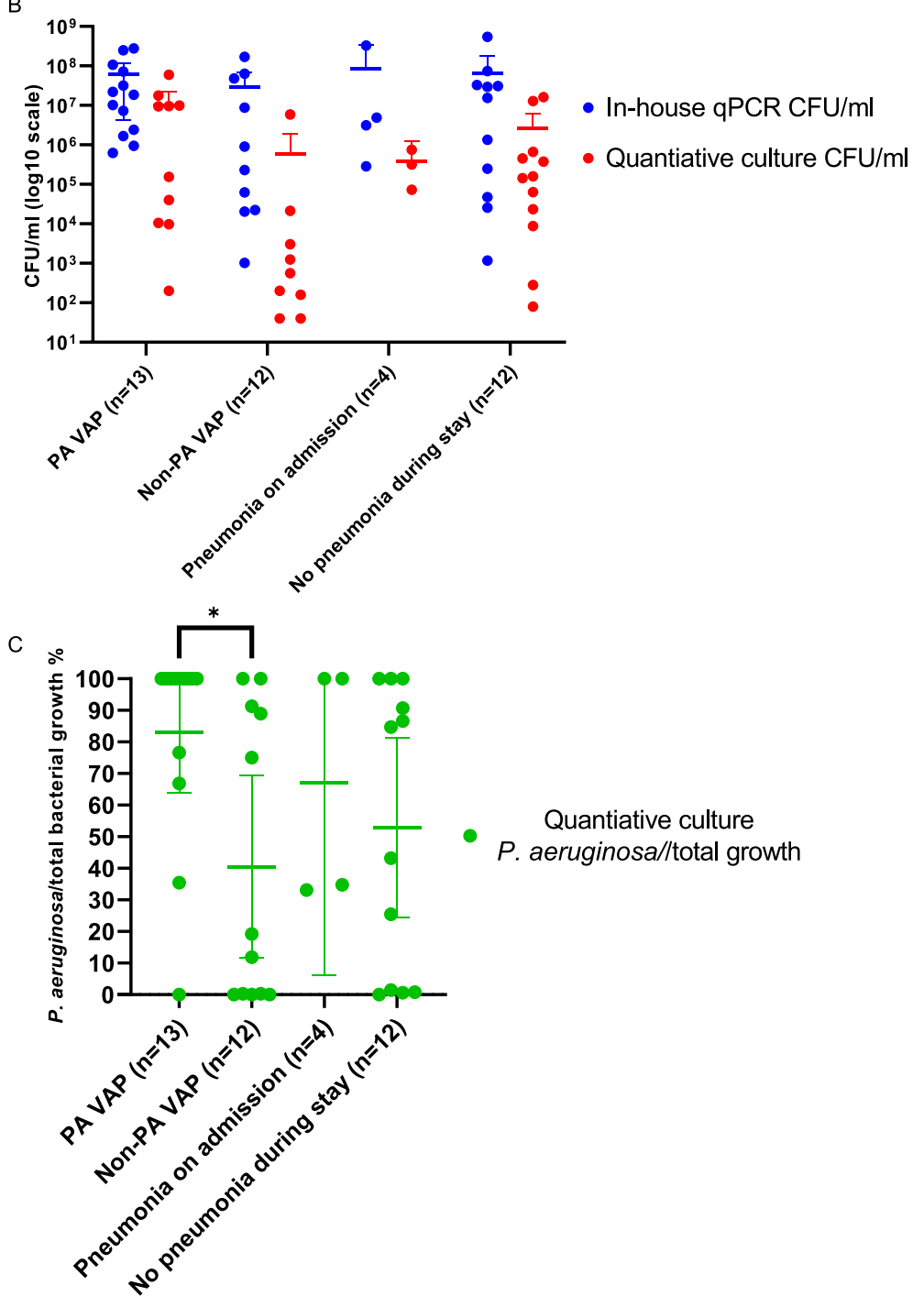

Fig. 3 (See legend on previous page.) 
PCR-based Ct values (Fig. 2A). Samples from both PA and non-PA VAP showed overlaps in Ct values for both the in-house qPCR and the GeneXpert PA assay. Although $61.5 \%$ and $53.8 \%$ of PA VAP diagnosed patients showed Ct values of $<26.3$ and $<21.4$ with the GeneXpert PA assay and in-house qPCR respectively, the samples from non-PA VAP also showed Ct values below those ranges however the percentages were lower (25\% for both the GeneXpert PA assay and the in-house qPCR). The receiver operating characteristic curve (Additional file 1: Figure S1) shows that the GeneXpert is useful yet would result in over-diagnosis of PA VAP. Further, the currently used method, semi-quantitative culture, has the advantage of detecting other co-existing pathogens in ETA samples. As seen in some of the VAP patients included in our study, $P$. aeruginosa was detected however VAP was attributed to another pathogen that was present at an even higher abundance.

Although all methods showed good sensitivity, none was $100 \%$ sensitive compared to the extended gold standard. In the enrichment cultures and qPCR, four and three samples, respectively, were negative while they were positive by the extended gold standard method. The BHI enrichment culture was performed using leftover sample after quantitative culture and in-house qPCR which might have resulted in too small of an inoculum, as indicated by the negative blood agar plates after enrichment. Two false negative samples in the qPCR had low loads as confirmed by semi-quantitative and quantitative culture and one was also negative with the GeneXpert PA assay while the other had high $\mathrm{Ct}$ values. The third sample had a moderate load in semi-quantitative culture which was reflected in both the GeneXpert PA assay and the quantitative culture. A possible explanation for the negative result might be a low homogeneity of the sample. The results of the enrichment cultures are counter intuitive since a high sensitivity is to be expected and a lower specificity. Sample 31 might be affected by the heterogeneity of the sample since both the quantitative and enrichment culture were falsely negative. The other assays showed that not only $P$. aeruginosa was present and it might have been outcompeted by other bacteria in the BHI broth.

However, the GeneXpert PA assay provides a fast and accurate method highlighting its potential as a screening assay to detect $P$. aeruginosa in mechanically ventilated patients to take forward preventive strategies that require early and rapid detection of $P$. aeruginosa colonization.

\section{Conclusion}

$P$. aeruginosa is a common cause of VAP, a frequent nosocomial infection. Rapid and accurate detection of lower respiratory tract colonization and/or infection with $P$. aeruginosa may inform targeted preventive and therapeutic strategies. The GeneXpert PA assay is a recently introduced molecular test directly detecting $P$. aeruginosa in ETA samples with an average turnaround time of $60 \mathrm{~min}$. We show here for the first time that the GeneXpert PA assay is a rapid and sensitive method for direct detection of $P$. aeruginosa in the ETA samples with $97.6 \%$ sensitivity and $100 \%$ specificity in comparison to the extended gold standard method.

\section{Abbreviations}

ETA: Endotracheal aspirates; VAP: Ventilator-associated pneumonia; ICU: Intensive care unit; qPCR: Quantitative TaqMan real-time PCR; Ct: Cyclic threshold; CFU: Colony-forming units; $\mathrm{BHI}$ : Brain heart infusion.

\section{Supplementary Information}

The online version contains supplementary material available at https://doi. org/10.1186/s13756-021-00978-9.

Additional file 1. Results from the five assays evaluated in this study.

\section{Acknowledgements}

We thank Anouk Vanderstraeten, Gert Leten, Liesbeth Bryssinck, Pien Barendsen, and Petra Vertongen (ICU) for excellent technical assistance.

\section{Authors' contributions}

This study was designed by AR, MTE, LY, MM, SK-S, HG, and SMK. Sample and clincial data collection was done by JC, LT, CL, VM, and PJ. Experimental work was done by JC, ATR, and LVH. Data were analysed and interpreted by TEvdS, JC, LVH, MM, SKS, and SMK. Statistical analysis was performed by TEvdS, LY, and SKS. The manuscript was drafted by TEVdS, JC, LT, MB and SMK, and was edited by all authors. All authors read and approved the final manuscript.

\section{Funding}

The research leading to these results has received support from the Innovative Medicines Initiative Joint Undertaking, COMBACTE-NET (Combatting bacterial resistance in Europe-Networks) and COMBACTE-MAGNET (Combatting bacterial resistance in Europe-Molecules against Gram-negative infections) under Grant Agreement $n^{\circ} 115523$ and 115737 resources of which are composed of financial contribution from the European Union Seventh Framework Programme (FP7/2007-2013) and EFPIA companies in kind contribution. TEvdS, $\mathrm{MB}$, and BBX were supported by COMBACTE-MAGNET. JC was supported by the University of Antwerp doctoral assistant funds. LT was supported by COMBACTE-NET. The sponsors of the study had no role in study design, data collection, data analysis, data interpretation, or writing the report. The corresponding author had full access to all the data in the study and had final responsibility for the decision to submit for publication.

Availability of data and materials

Please contact the author for data requests.

\section{Declarations}

Ethical approval and consent to participate

All data were retrieved from the patient data management system (Metavision, iMDsoft, Düsseldorf, Germany). The study was reviewed and approved by the hospital's institutional review board (Number 11/2/19 and 16/28/298). Since all data were fully de-identified, the necessity of obtaining informed consent was waived.

Consent of publication

Not applicable. 


\section{Competing interests}

The authors declare that they have no competing interests.

\section{Author details}

'Laboratory of Medical Microbiology, Vaccine and Infectious Disease Institute, University of Antwerp, Wilrijk, Belgium. ${ }^{2}$ Laboratory of Clinical Microbiology, Antwerp University Hospital, Edegem, Belgium. ${ }^{3}$ Microbial Sciences, BioPharmaceuticals R\&D, AstraZeneca, Gaithersburg, USA. ${ }^{4}$ Statistical Sciences, BioPharmaceuticals R\&D, AstraZeneca, Gaithersburg, USA. ${ }^{5}$ Early-Stage Development, Cardiovascular, Renal and Metabolism, BioPharmaceuticals R\&D, AstraZeneca, Gaithersburg, USA. ${ }^{6}$ Department of Intensive Care Medicine, Antwerp University Hospital, Edegem, Belgium. ${ }^{7}$ Molecular Pathology Group, Cell Biology and Histology, University of Antwerp, Wilrijk, Belgium.

Received: 19 March 2021 Accepted: 8 July 2021

Published online: 23 July 2021

\section{References}

1. Moradali MF, Ghods S, Rehm BHA. Pseudomonas aeruginosa lifestyle: a paradigm for adaptation, survival, and persistence. Front Cell Infect Microbiol. 2017;7:39

2. Paling FP, Wolkewitz M, Depuydt P, de Bus L, Sifakis F, Bonten MJM, et al. P. aeruginosa colonization at ICU admission as a risk factor for developing $P$. aeruginosa ICU pneumonia. Antimicrob Resist Infect Control. 2017;6:38.

3. Kalanuria A, Zai W, Mirski M. Ventilator-associated pneumonia in the ICU. Crit Care. 2014;18:208.

4. Chastre J, Fagon J-Y. Ventilator-associated pneumonia. Am J Respir Crit Care Med. 2002;165:867-903.

5. Boldin B, Bonten MJM, Diekmann O. Relative effects of barrier precautions and topical antibiotics on nosocomial bacterial transmission: results of multi-compartment models. Bull Math Biol. 2007:69:2227-48.

6. Brusselaers N, Labeau S, Vogelaers D, Blot S. Value of lower respiratory tract surveillance cultures to predict bacterial pathogens in ventilatorassociated pneumonia: systematic review and diagnostic test accuracy meta-analysis. Intensive Care Med. 2013;39:365-75.
7. Jorens PG. Sticking to an old definition of ventilator-associated pneumonia is not old-fashioned. Respir Care. 2016;61:390-2.

8. Garcia LS, Isenberg HD. Clinical microbiology procedures handbook. Washington, DC: American Society for Microbiology Press; 2010.

9. Choi HJ, Kim MH, Cho MS, Kim BK, Kim JY, Kim C, et al. Improved PCR for identification of Pseudomonas aeruginosa. Appl Microbiol Biotechnol. 2013:97:3643-51.

10. Fey A, Eichler S, Flavier S, Christen R, Höfle MG, Guzmán CA. Establishment of a real-time PCR-based approach for accurate quantification of bacterial RNA targets in water, using Salmonella as a model organism. Appl Environ Microbiol. 2004;70:3618-23.

11. Sharpe JP, Magnotti LJ, Weinberg JA, Swanson JM, Schroeppel TJ, Clement LP, et al. Adherence to an established diagnostic threshold for ventilator-associated pneumonia contributes to low false-negative rates in trauma patients. J Trauma Acute Care Surg. 2015;78:468-74.

12. Ali SO, Yu XQ, Robbie GJ, Wu Y, Shoemaker K, Yu L, et al. Phase 1 study of MEDI3902, an investigational anti-Pseudomonas aeruginosa PcrV and Psl bispecific human monoclonal antibody, in healthy adults. Clin Microbiol Infect. 2019;25:629.e1-629.e6.

13. Coppens J, Van Heirstraeten L, Ruzin A, Yu L, Timbermont L, Lammens C et al. Comparison of GeneXpert MRSA/SA ETA assay with semi-quantitative and quantitative cultures and nuc gene-based qPCR for detection of Staphylococcus aureus in endotracheal aspirate samples. Antimicrob Resist Infect Control. 2019;8:4.

14. Eroshenko D, Polyudova T, Korobov V. N-acetylcysteine inhibits growth, adhesion and biofilm formation of Gram-positive skin pathogens. Microb Pathog. 2017;105:145-52.

15. Weiser R, Donoghue D, Weightman A, Mahenthiralingam E. Evaluation of five selective media for the detection of Pseudomonas aeruginosa using a strain panel from clinical, environmental and industrial sources. J Microbiol Methods. 2014;99:8-14.

\section{Publisher's Note}

Springer Nature remains neutral with regard to jurisdictional claims in published maps and institutional affiliations.
Ready to submit your research? Choose BMC and benefit from:

- fast, convenient online submission

- thorough peer review by experienced researchers in your field

- rapid publication on acceptance

- support for research data, including large and complex data types

- gold Open Access which fosters wider collaboration and increased citations

- maximum visibility for your research: over 100M website views per year

At $B M C$, research is always in progress.

Learn more biomedcentral.com/submissions 